\title{
Two Extensions of Data Assimilation by Field Alignment $^{\star}$
}

\author{
Sai Ravela \\ Earth, Atmospheric and Planetary Sciences \\ Massachusetts Institute of Technology \\ ravela@mit.edu
}

\begin{abstract}
Classical formulations of data-assimilation perform poorly when forecast locations of weather systems are displaced from their observations. They compensate position errors by adjusting amplitudes, which can produce unacceptably "distorted" states. Motivated by cyclones, in earlier work we show a new method for handling position and amplitude errors using a single variational objective. The solution could be used with either ensemble or deterministic methods. In this paper, extension of this work in two directions is reported. First, the methodology is extended to multivariate fields commonly used in models, thus making this method readily applicable. Second, an application of this methodology to rainfall modeling is presented.
\end{abstract}

\section{Introduction}

Environmental data assimilation is the methodology for combining imperfect model predictions with uncertain data in a way that acknowledges their respective uncertainties. It plays a fundamental role in DDDAS. However, data assimilation can only work when the estimation process properly represents all sources of error. The difficulties created by improperly represented error are particularly apparent in mesoscale meteorological phenomena such as thunderstorms, squall-lines, hurricanes, precipitation, and fronts. Errors in mesoscale models can arise in many ways but they often manifest themselves as errors in the position. We typically cannot attribute position error to a single or even a small number of sources and it is likely that the position errors are the aggregate result of errors in parameter values, initial conditions, boundary conditions and others. In the context of cyclones operational forecasters resort to ad hoc procedures such as bogussing [4] . A more sophisticated alternative is to use data assimilation methods. Unfortunately, sequential [10], ensemble-based [9] and variational [12]3] state estimation methods used in data assimilation applications adjust amplitudes to deal with position error. Adjusting amplitudes doesn't really fix position error, and instead, can produce unacceptably distorted estimates.

In earlier work [16], we show how the values predicted at model grid points can be adjusted in amplitude and moved in space in order to achieve a better fit to observations. The solution is general and applies equally well to meteorological, oceanographic, and

\footnotetext{
* This material is supported NSF CNS 0540259.
} 
hydrological applications. It involves solving two equations, in sequence, described as follows:

Let $X=X(\mathbf{r})=\left\{X\left[\underline{r}_{1}^{T}\right] \ldots X\left[\underline{r}_{m}^{T}\right]\right\}$ be the model-state vector defined over a spatially discretized computational grid $\Omega$, and $\mathbf{r}^{\mathrm{T}}=\left\{\underline{r}_{i}=\left(x_{i}, y_{i}\right)^{T}, i \in \Omega\right\}$ be the position indices. Similarly, let $\mathbf{q}$ be a vector of displacements. That is, $\mathbf{q}^{\mathbf{T}}=\left\{\underline{q}_{i}=\right.$ $\left.\left(\Delta x_{i}, \Delta y_{i}\right)^{T}, i \in \Omega\right\}$, and $X(\mathbf{r}-\mathbf{q})$ represents displacement of $X$ by $\mathbf{q}$. The displacement field $\mathbf{q}$ is real-valued, so $X(\mathbf{r}-\mathbf{q})$ must be evaluated by interpolation if necessary.

We wish to find $(X, \mathbf{q})$ that has the maximum a posteriori probability in the distribution $P(X, \mathbf{q} \mid Y)$, where $Y$ is the observation vector. Using Bayes rule we obtain $P(X, \mathbf{q} \mid Y) \propto P(Y \mid X, \mathbf{q}) P(X \mid \mathbf{q}) P(\mathbf{q})$. Assume a linear observation model with uncorrelated noise in space and time, the component densities to be Gaussian and the displacement field solution is smooth and non-divergent. Then, the following EulerLagrange equations are obtained and solved sequentially.

1) Alignment. Define $\mathbf{p}=\mathbf{r}-\mathbf{q}$ and the alignment equation is then written at each grid node $i$ as:

$$
w_{1} \nabla^{2} \underline{q}_{i}+w_{2} \nabla\left(\nabla \cdot \underline{q}_{i}\right)+\left[\left.\nabla X^{T}\right|_{\mathbf{p}} H^{T} R^{-1}(H[X(\mathbf{p})]-Y)\right]_{i}=0
$$

Equation[1introduces a forcing based on the residual between fields. The constraints on the displacement field allow the forcing to propagate to a consistent solution. Equation 1 is non-linear, and is solved iteratively.

2) Amplitude Adjustment: The aligned field $X(\hat{\mathbf{p}})$ is used in the second step for a classical Kalman update:

$$
\hat{X}(\hat{\mathbf{p}})=X(\hat{\mathbf{p}})+B_{\hat{Q}} H^{T}\left(H B_{\hat{Q}} H^{T}+R\right)^{-1}(Y-H X(\hat{\mathbf{p}}))
$$

The covariance $B_{\hat{Q}}$ is computed after alignment. It can be estimated using ensembles (when each has been aligned), or any method that would otherwise be used on the field $X$.

\section{Multivariate Formulation}

To be applicable in practice, the field alignment algorithm must be extended to the multivariate case, including vector fields. As an example, consider 2D fields (3D-fields can be handled analogously), say two components of velocity and pressure. Now partition the state $X$ and observations $Y$ into component fields, $P, U, V$ for pressure. To align $X$ to $Y$, we constrain individual fields to have the same displacements. Displacing vector fields, however, involves the Jacobian of the deformation ( when the wind field rotates, both the coordinate and wind-field components rotate). Therefore, if $\psi$ is a scalar function undergoing a deformation $\psi(\mathbf{r}-\mathbf{q})$, then the gradient vector field undergoes a transformation that is expressed as $\left.(\nabla \mathbf{q})^{T} \nabla \psi\right|_{\mathbf{r}-\mathbf{q}}$. We introduce, therefore, for the wind velocity components, the variables $\tilde{U}, \tilde{V}$, defined as

$$
\left(\begin{array}{c}
\tilde{U}_{i} \\
\tilde{V}_{i}
\end{array}\right)=\left(\nabla \underline{q}_{i}\right)^{T}\left(\begin{array}{c}
U_{i} \\
V_{i}
\end{array}\right)
$$


The field alignment equation now looks as follows:

$$
\begin{aligned}
w_{1} \nabla^{2} \underline{q}_{i}+w_{2} \nabla\left(\nabla \cdot \underline{q}_{i}\right) & +\left\{\left[\left.\nabla P^{T}\right|_{\mathbf{r}-\mathbf{q}} H_{P}^{T} R_{P}^{-1}\left(H_{P} P(\mathbf{r}-\mathbf{q})-Y_{P}\right)\right\}_{i}\right. \\
& +\left\{\left[\left.\nabla \tilde{U}^{T}\right|_{\mathbf{r}-\mathbf{q}} H_{U}^{T} R_{U}^{-1}\left(H_{U} \tilde{U}(\mathbf{r}-\mathbf{q})-Y_{U}\right)\right\}_{i}\right. \\
& +\left\{\left[\left.\nabla \tilde{V}^{T}\right|_{\mathbf{r}-\mathbf{q}} H_{V}^{T} R_{V}^{-1}\left(H_{V} \tilde{V}(\mathbf{r}-\mathbf{q})-Y_{V}\right)\right\}_{i}=0\right.
\end{aligned}
$$

Here $R$ is the observation noise covariance, $H_{P}, H_{U}, H_{V}$ are the observation operators, and $Y_{U}, Y_{V}, Y_{P}$ are the component fields of the observation vector. We demonstrate the use of the 2-step method on multivariate fields next.

Example: An example is now demonstrated using pressure and velocity. Figures 1 contain "filled" iso-contour plots of state fields. The first guess fields shown down the first column is rotated by $40^{\circ}$ from truth (shown in second column). Measurements are made at every $5^{\text {th }}$ pixel on truth, marked in white dots or black crosses in third column. There is an amplitude error of a multiplicative factor 1.1, the observational noise, $30 \%$ of peak amplitude in pressure, and $7 \%$ in velocity components, i.i.d. The background error covariance (modeled isotropic, see [16] for flow dependent results) in this example is substantially more uncertain than the observational uncertainty.

The fourth column of Figure 1 depicts the analysis of 3DVAR-only for pressure and velocity components. The pressure and velocity fields are clearly "smeared". In contrast, the rightmost column of Figure 1 depicts a much better analysis when field alignment is used as the preprocessing step to 3DVAR. Note that the first guess and observations fed to 3DVAR-only and field alignment algorithm is the same.

In Figure 2, using the nonlinear balance equation, we compare the analyzed and diagnosed pressure fields to study how well-balanced alignment is. Result: the alignment followed by 3DVAR preserves the balance to a far greater degree than 3DVAR alone, see Figure 2

Figure 3 compares analysis and balance errors for various cases of sparsity. The $\mathrm{x}-$ axis of the left panel depicts sparsity; 1 implies every location was observed, 10 implies every $10^{t h}$ location was observed. The y-axis represents normalized error, normalized by the maximum pressure differential. The left panel's bar charts contain filled bars comparing the analyzed pressure vs. truth, using open bars for 3DVAR and closed ones for field alignment followed by 3DVAR. We can see that as the observations get more sparse, 3DVAR performance degrades more sharply than a field alignment preprocessed version.

The right panel of Figure 3 compares the analyzed and diagnosed pressure, with the $\mathrm{x}$-axis representing sparsity and the $\mathrm{y}$-axis, normalized error. The differences rise sharply for 3DVAR-only and, in fact, after about a sparsity of 6 pixels, 3DVAR-only breaks down. The analysis does not compensate for position error and this is clearly seen in the analysis errors shown in the right panel corresponding to this case. Therefore, although the diagnosed and analyzed pressure fields in 3DVAR-only find themselves in good agreement, they are quite far away from the truth! In contrast, compensating position error using field alignment yields analysis and balance errors that are much smaller. They do grow, but much more slowly as function of observational sparsity. 


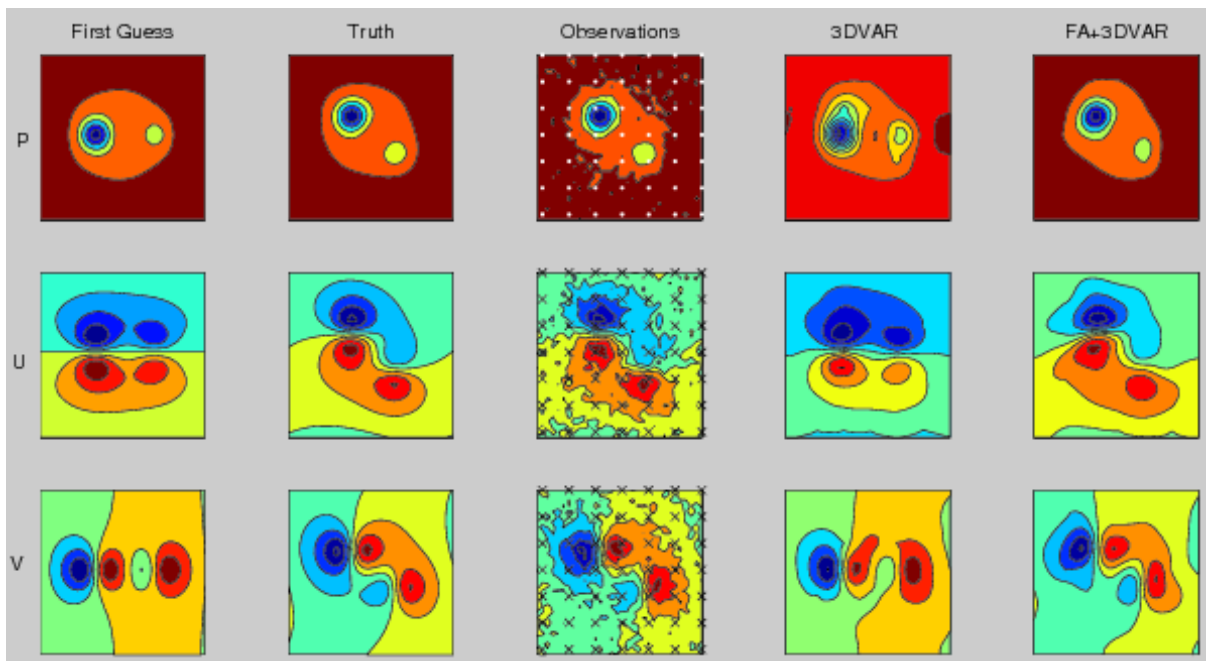

Fig. 1. The left column of panels is the first guess, the second column truth, the third shows observations, taken at indicated locations, the fourth shows 3DVAR-only analysis and the rightmost shows field alignment followed by 3DVAR with identical truth, first guess and observations. The top row corresponds to pressure, the second row to U component of velocity and the third column is the $\mathrm{V}$ component.

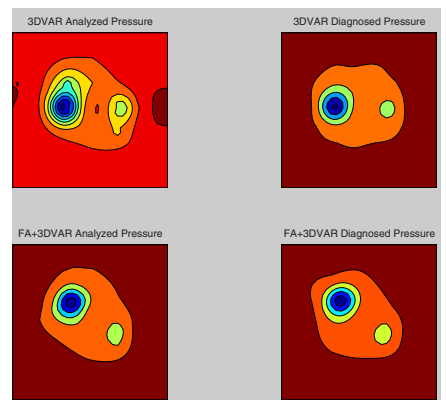

Fig. 2. This figure depicts a comparison of balance between 3DVAR and our approach. The analyzed and diagnosed 3DVAR pressures (top two panels) are substantially different than the corresponding pressure fields using 3 DVAR after alignment.

\section{Application to Velocimetry}

In contrast to hurricanes, we apply the methodology to rainfall modeling. Rainfall models broadly fall into two categories. The first is a meteorological or the quantitative precipitation forecasting model, such as Mesoscale Model (MM5) [2], the stepmountain Eta coordinate model [1], and the Regional Atmospheric Modeling System (RAMS) [5], etc. The second type is the spatiotemporal stochastic rainfall model. It aims to summarize the spatial and temporal characteristics of rainfall by a small set of 

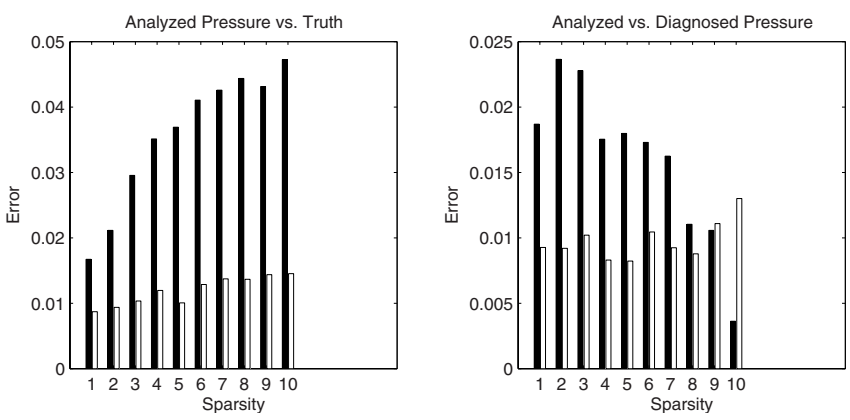

Fig. 3. The x-axis of these graphs represents sparsity. The y-axis of the left panel shows the normalized error between the analyzed pressure and truth, and the right panel shows the normalized error between analyzed and diagnosed pressure. The filled bars depict the 3DVAR-only case, and the open bars are for field alignment followed by 3DVAR.
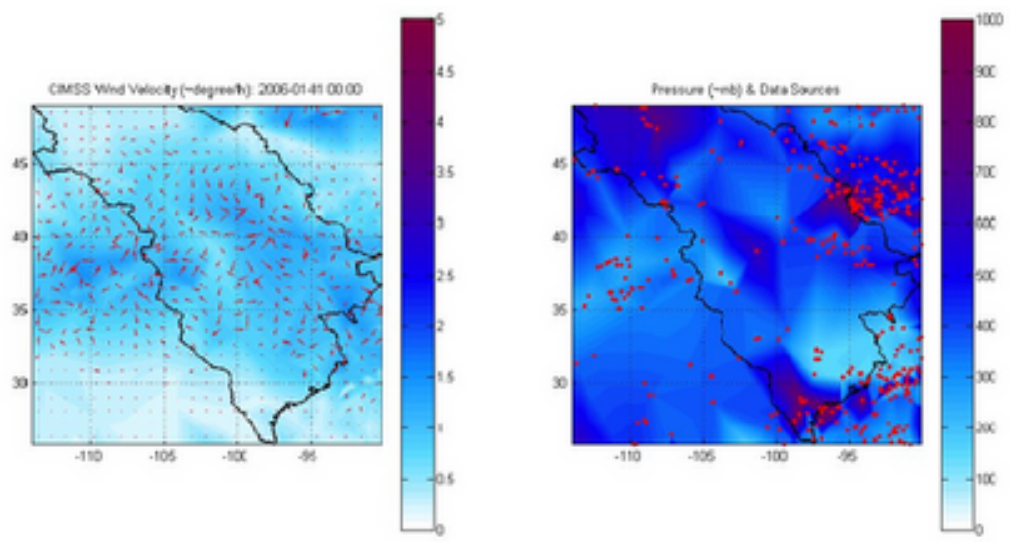

Fig. 4. CIMSS Winds derived from GOES data at 2006-04-06-09Z (left) and pressure (right). The velocity vectors are sparse and contain significant divergence.

parameters [6]. This type of model usually simulates the birth and decay of rain-cells and evolve them through space and time using simple physical descriptions. Despite significant differences among these rainfall models, the concept of propagating rainfall through space and time are relatively similar.

The major ingredient required to advect rainfall is a velocity field. Large spatial-scale (synoptic) winds are inappropriate for this purpose for a variety of reasons. Ironically, synoptic observations can be sparse to be used directly and although synoptic-scale wind analyses produced from them (and models) do produce dense spatial estimates, such estimates often do not contain variability at the meso-scales of interest. The motion of mesoscale convective activity is a natural source for velocimetry. Indeed, there exist products that deduce "winds" by estimating the motion of temperature, vapor and other fields evolving in time [7]8]. 

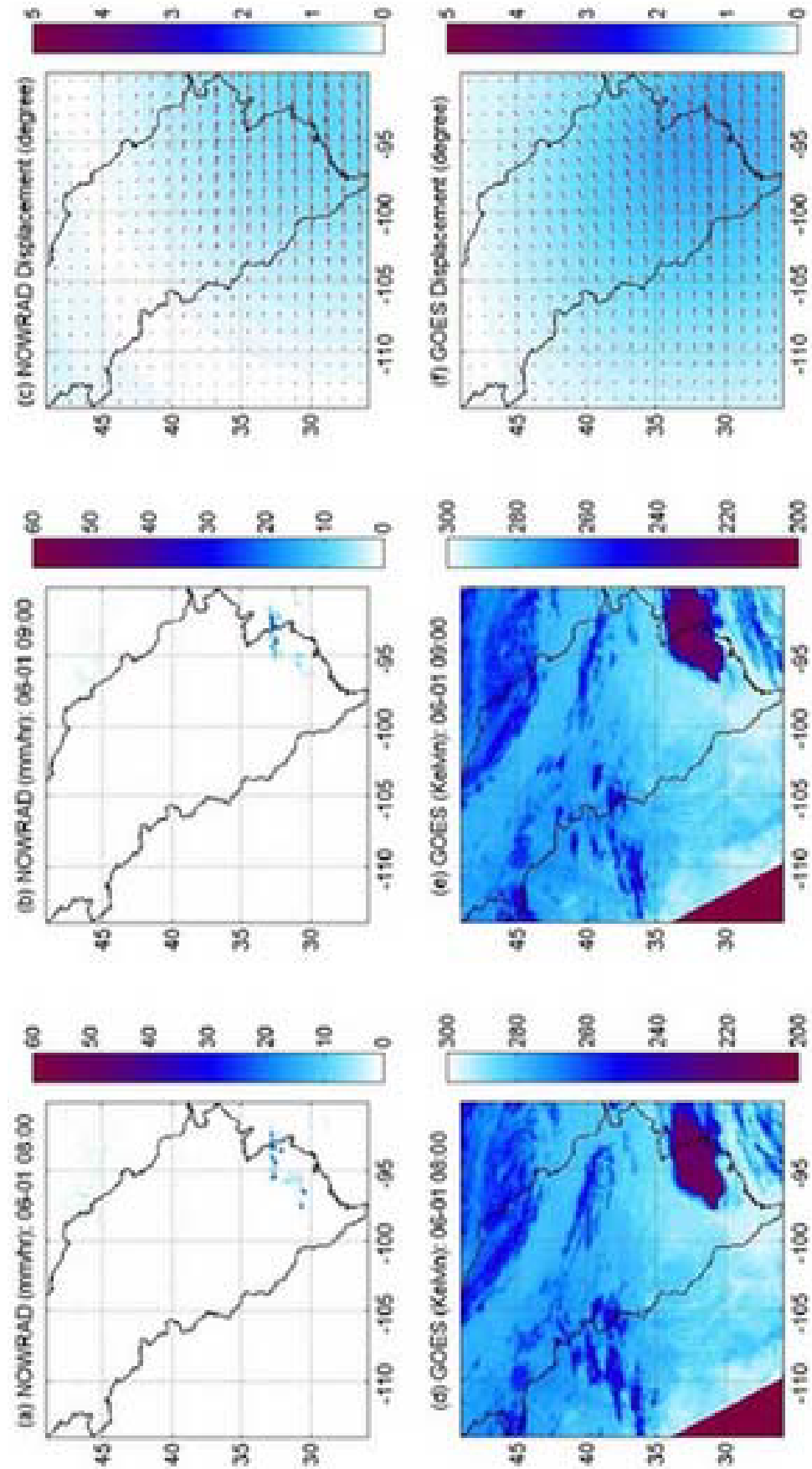

Fig. 5. Deriving velocimetry information from satellite observations, Nexrad (top), GOES (bottom). See text for more information. 
In this paper, we present an algorithm for velocimetry from observed motion from satellite observations such as GOES, AMSU, TRMM, or radar data such as NOWRAD. This is obtained by the direct application of equation 1 to two time separated images. This approach provides marked improvement over other methods in conventional use. In contrast to correlation based approaches used for deriving velocity from GOES imagery, the displacement fields are dense, quality control is implicit, and higher-order and smallscale deformations can be easily handled. In contrast with optic-flow algorithms [13 11], we can produce solutions at large separations of mesoscale features, at large time-steps or where the deformation is rapidly evolving. A detailed discussion is presented in [15].

Example: The performance of this algorithm is illustrated in a velocimetry computation. To compare, we use CIMSS wind-data satellite data [8], depicted in Figure 4 obtained from CIMSS analysis on 2006-06-04 at 09Z. CIMSS wind-data is shown over the US great plains, and were obtained from the sounder. The red dots indicate the original location of the data. The left subplot shows wind speed (in degree/hr). The right ones show pressure, and the location of raw measurements in red.

It can be seen in the map in Figure 4 that the operational method to produce winds generate sparse vectors and, further, has substantial divergence. Considering the lengthscales, this isn't turbulence and wind vectors are more likely the result of weak quality control. A more detailed discussion is presented in [15].

In contrast, our method produces dense flow fields, and quality control is implicit from regularization constraints. Figure $5(\mathrm{a}, \mathrm{b})$ shows a pair of NOWRAD images at 2006-06-01-0800Z and 2006-06-01-0900Z respectively, and the computed flow field in Figure 5(c). Similarly, Figure 5(d,e,f) show the GOES images and velocity from the same time frame over the deep convective rainfall region in the Great Plains example. The velocities are in good agreement with CIMSS derived winds where magnitudes are concerned, but the flow-fields are smooth and visual confirmation of the alignment provides convincing evidence that they are correct.

\section{Discussion and Conclusions}

The joint position-amplitude assimilation approach is applicable to fields with coherent structures. Thus, problems in reservoir modeling, convection, rainfall modeling, tracking the ocean and atmosphere will benefit. The solution to the assimilation objective can be computed efficiently in two steps: diffeomorphic alignment followed by amplitude adjustment. This solution allows ready use with existing methods, making it an attractive option for operational practice.

The alignment formulation does not require features to be identified. This is a significant advantage in sparse observations when features cannot be clearly delineated. The alignment formulation can be extended easily to multivariate fields and can be used for a variety of velocimetry problems including particle image velocimetry, velocity from tracer-transport, and velocity from GOES and other satellite data. In relation to GOES, our approach implicitly provides quality control in terms of smoothness, and produces dense displacement fields.

To complete this body of work on position-amplitude estimation, we are conducting research in the following directions: 
1. We recently demonstrated [14] that an ensemble filter can be developed when both observations and states have position and amplitude error. This situation occurs in the context of rainfall models, where both satellite derived rain cells and model forecast cells contain position and amplitude error.

2. The position-amplitude smoother: We develop an optimal fixed-interval and fixed-lag ensemble smoother [17]. Our results show that fixed-interval ensemble smoothing is linear in the interval and fixed-lag is independent of lag length. We are extending this smoother to the position-amplitude problem.

3. New constraints: The smoothness constraint has been observed to provide weak control in certain problems. In ongoing work, we have reformulated the alignment problem using a spectral constraint on the deformation field.

\section{References}

1. T. L. Black. The new nmc moesoscale eta model: Description and forecast examples. Weather and Forecasting, 9(2):265-278, 1994.

2. F. Chen and J. Dudhia. Coupling an advanced land surface-hydrology model with the penn state-ncar mm5 modeling system. part i: Model implementation and sensitivity. Monthly Weather Review, 129(4):569-585, 2001.

3. P. Courtier. Variational methods. J. Meteor. Soc. Japan, 75, 1997.

4. C. Davis and S. Low-Nam. The ncar-afwa tropical cyclone bogussing scheme. Technical Memorandum, Air Force Weather Agency (AFWA), Omaha, NE, [http://www.mmm.ucaredu/ mm5/mm5v3/tc-report.pdf], 2001.

5. A. Orlandi et al. Rainfall assimilation in rams by means of the kuo parameterisation inversion: Method and preliminary results. Journal of Hydrology, 288(1-2):20-35, 2004.

6. C. Onof et al. Rainfall modelling using poisson-cluster processes: A review of developments. Stochastic Environmental Research and Risk Assessment, 2000.

7. C. S. Velden et al. Upper-tropospheric winds derived from geostationary satellite water vapor observations. Bulletin of the American Meteorological Society, 78(2):173-195, 1997.

8. C. Velden et al. Recent innovations in deriving tropospheric winds from meteorological satellites. Bulletin of the American Meteorological Society, 86(2):205-223, 2005.

9. G. Evensen. The ensemble kalman filter: Theoretical formulation and practical implementation. Ocean Dynamics, 53:342-367, 2003.

10. A. Gelb. Applied Optimal Estimation. MIT Press, 1974.

11. D. J. Heeger. Optical flow from spatiotemporal filters. International Journal of Computer Vision, pages 279-302, 1988.

12. A. C. Lorenc. Analysis method for numerical weather predictin. Q. J. R. Meteorol. Soc., 112:1177-1194, 1986.

13. H.-H Nagel. Displacement vectors derived from second order intensity variations in image sequences. Computer Vision, Graphics and Image Processing, 21:85-117, 1983.

14. S. Ravela and V. Chatdarong. How do we deal with position errors in observations and forecasts? In European Geophysical Union Annual Congress, 2006.

15. S. Ravela and V. Chatdarong. Rainfall advection using velocimetry by multiresolution viscous alignment. Technical report, arXiv, physics/0604158, April 2006.

16. S. Ravela, K. Emanuel, and D. McLaughlin. Data assimilation by field alignment. Physica D (Article in Press), doi:10.1016/j.physd.2006.09.035, 2006.

17. S. Ravela and D. McLaughlin. Fast ensemble smoothing. Ocean Dynamics(Article in Press), DOI 10.1007/s10236-006-0098-6, 2007. 